\title{
Avaliação das capacidades de vigilância do Centro de Informações Estratégicas em Vigilância em Saúde do Ministério da Saúde, Brasil
}

\section{Assessment of surveillance capacities of the Center for Strategic Information in Health Surveillance, Ministry of Health, Brazil}

\section{Evaluación de las capacidades de vigilancia del Centro de Informaciones Estratégicas de Vigilancia en Salud del Ministerio de Salud, Brasil}

\author{
Melina Érica Santos ${ }^{1}$ \\ José Fernando de Souza Verani² \\ Vitor Laerte Pinto Junior ${ }^{3}$ \\ Wildo Navegantes de Araújo ${ }^{4}$
}

RESUMO: O Centro de Informações Estratégicas em Vigilância em Saúde da Secretaria de Vigilância em Saúde do Ministério da Saúde (CIEVS/MS) foi institucionalizado no contexto do Regulamento Sanitário Internacional (RSI) como a estratégia de vigilância das emergências em saúde pública, a fim de desenvolver os processos de detecção, avaliação e notificação das potenciais emergências em saúde pública de importância nacional e internacional, na representação do Ponto Focal Nacional do Brasil para o RSI. O objetivo deste artigo é avaliar as capacidades de vigilância das emergências em saúde pública pelo CIEVS/MS. O método utilizado foi a pesquisa avaliativa com a aplicação do modelo lógico, segundo o qual foram estruturados os indicadores

\footnotetext{
1 Mestrado Profissional em Saúde Pública (Saúde Global e Diplomacia da Saúde) pela Escola Nacional de Saúde Pública Sergio Arouca, Brasil(2014); Analista Técnico de Políticas Sociais do Ministério da Saúde .

2 Doutorado em Ciências na Área de Saúde Pública pela Escola Nacional de Saude Publica, Brasil(2005). Pesquisador titular-U Ref/Niv. III da Fundação Oswaldo Cruz .

3 Doutorado em Medicina Tropical pela Fundação Oswaldo Cruz, Brasil(2008)

Pesquisador Titular da Fundação Oswaldo Cruz Brasília

4 Doutorado em Biotecnologia em Saúde e Medicina Investigativa pela Fundação Oswaldo Cruz, Brasil(2011)

Professor adjunto da Universidade de Brasília.
} 
para avaliar os processos de trabalho do CIEVS/MS, considerando os aspectos político, técnico e organizacional, nos componentes de detecção, avaliação e notificação. A classificação das capacidades de vigilância do CIEVS/MS foi parcialmente adequada, identificando potencialidades e fragilidades da estratégia. O modelo lógico revelou-se como método importante para avaliação do serviço de vigilância em saúde. Dessa forma, recomenda-se que os processos de trabalho do CIEVS/MS sejam sistematizados e fortalecidos para a adequada vigilância das emergências em saúde pública no Brasil.

Palavras-chave: Vigilância Epidemiológica. Notificação. Emergências. Saúde Pública.

ABSTRACT: The Center for Strategic Information in Health Surveillance of the Health Surveillance Secretariat, Ministry of Health, Brazil (CIEVS/MS) was institutionalized in the context of the International Health Regulations (IHR) as the strategy for surveillance of public health emergencies, with the objective to ensure detection, evaluation and reporting of public health events that may constitute public health emergencies of national and international concern, representing the National IHR Focal Point of Brazil. The purpose of this paper is to assess the surveillance capacities of CIEVS/MS. The applied method was the evaluation research using the logic model, whereby the indicators were formulated to evaluate CIEVS/MS work process, according to political, technical and institutional aspects in detection, evaluation and notification components. The surveillance capacities were classified as partially appropriate, revealing the strengths and weaknesses of CIEVS/MS work process. The logic model was an important method to evaluate the health surveillance service. Thus, it is recommended that CIEVS/MS work process be organized and strengthened for proper monitoring of public health emergencies in Brazil.

Keywords: Epidemiological Surveillance. Notice. Emergencies. Public Health.

RESUMEN: El Centro de Informaciones Estratégicas de Vigilancia en Salud de la Secretaria de Vigilancia en Salud del Ministerio de Salud de Brasil (CIEVS/MS) fue institucionalizado en el contexto del Reglamento Sanitario Internacional (RSI) como la estrategia de vigilancia de las emergencias de salud pública, con la finalidad de desarrollar los procesos de detección, evaluación y notificación de las potenciales emergencias de salud pública de importancia nacional e internacional, en representación al Punto Focal Nacional de Brasil para el RSI. El objetivo de este artículo es el de analizar las capacidades de vigilancia de las emergencias de salud pública por el CIEVS/MS. El método utilizado fue la investigación evaluativa con aplicación del modelo lógico, según el cual los indicadores fueron estructurados para evaluar los procesos de trabajo del CIEVS/MS, considerando los aspectos político, técnico y organizacional, según los componentes de detección, evaluación y notificación. La clasificación de las capacidades de vigilancia del CIEVS/MS fue parcialmente adecuada, identificando potencialidades y fragilidades de la estrategia. El modelo lógico se demostró como un método importante para la evaluación del servicio de vigilancia en salud. De esa manera se recomienda que los procesos de trabajo del CIEVS/MS sean sistematizados y fortalecidos para la adequada vigilancia de las emergencias de salud pública en Brasil.

Palabras clave: Vigilancia Epidemiológica. Notificación. Urgencias Médicas. Salud Pública.

Tempus, actas de saúde colet, Brasília, 10(2), 219-234, jun, 2016.

ISSN 1982-8829 


\section{INTRODUÇÃO}

Historicamente, a vigilância vem sendo estruturada como função do Estado, tendo em vista o cumprimento das funções consideradas de interesse público ${ }^{1}$. A partir da década de 1950, a vigilância epidemiológica surge para designar as atividades de controle das doenças transmissíveis. No Brasil, a Campanha de Erradicação da Varíola é considerada o marco da institucionalização das ações de vigilância no país ${ }^{2}$.

No contexto do desenvolvimento da vigilância das doenças transmissíveis de importância internacional, foi consolidado o Regulamento Sanitário Internacional (RSI), cuja primeira versão de 1951 apresentou a finalidade de proteção dos países quanto à disseminação internacional de doenças. O RSI, aprovado em 2005 pela 58 a Assembleia Mundial da Saúde, está em vigor nos 194 países membros da Organização Mundial da Saúde (OMS), também denominados Estados Partes, desde 15 de junho de 2007. Segundo a Carta das Nações Unidas e os princípios do direito internacional, os Estados possuem o direito soberano de legislar e implementar a legislação a fim de cumprir suas próprias políticas de saúde, observando as determinações do Regulamento 3 .

O RSI de 2005 contempla novas estratégias de vigilância e controle das doenças transmissíveis, incluindo os eventos produzidos por agentes não biológicos. Além de representar a ampliação do escopo da vigilância para os eventos de saúde pública, destaca os critérios de avaliação de risco e a estratégia de uso do instrumento de decisão para a notificação de eventos ${ }^{4}$. Nesse sentido, orienta o estabelecimento de Pontos Focais Nacionais nos Estados Partes, permanentemente acessíveis para comunicação direta com os Pontos de Contato da OMS, nas situações de emergências em saúde pública de importância nacional e internacional ${ }^{5}$.

Os pontos focais são designados para facilitar a comunicação oportuna de informações de vigilância, sendo responsáveis pela notificação direta à contraparte da OMS nos termos do Regulamento, comunicação aos Pontos Focais Nacionais para o RSI dos demais países signatários e disseminação de informações às autoridades de saúde do seu próprio território. O Centro de Informações Estratégicas em Vigilância em Saúde do Ministério da Saúde (CIEVS/MS), institucionalizado por meio da Portaria $\mathrm{n}^{\mathrm{o}} 30$ de $2005^{6}$, é a unidade operacional do Ponto Focal Nacional do Brasil para o RSI.

Considerando a constante mudança nos padrões epidemiológicos e a necessidade de resposta oportuna aos agravos de importância em saúde pública, o CIEVS/MS reproduz a estratégia de vigilância baseada em eventos. De acordo com a OMS, a vigilância baseada em eventos se caracteriza pela detecção rápida de potenciais riscos à saúde pública a partir de informações oficiais, transmitidas pelos sistemas de notificação, ou não oficiais, veiculadas pela mídia, profissionais de saúde e população ${ }^{7}$.

Nesse sentido, o CIEVS/MS é responsável pela vigilância dos eventos de saúde pública de 
notificação compulsória imediata. As competências do CIEVS/MS envolvem a detecção, a avaliação, a notificação e o monitoramento dos riscos para a saúde pública e das emergências em saúde pública de importância nacional e internacional; além da comunicação oportuna das informações às unidades técnicas competentes do governo federal, aos Centros de Informações Estratégicas em Vigilância em Saúde das Secretarias de Saúde dos Estados (CIEVS/SES), das capitais (CIEVS/SMS) e do Distrito Federal, e aos Pontos Focais Nacionais para o RSI.

A Emergência em Saúde Pública de Importância Nacional (ESPIN) caracteriza-se por evento que representa risco para a saúde pública nacional. Segundo o Decreto $n^{\circ} 7.616$, a declaração de ESPIN ocorre nas situações de surtos ou epidemias, desastres ou desassistência à população, que extrapolem a capacidade de resposta da direção estadual do Sistema Único de Saúde (SUS) ou, especificamente nas situações epidemiológicas, "apresentem risco de disseminação nacional, sejam produzidos por agentes infecciosos inesperados, representem a reintrodução de doença erradicada ou apresentem gravidade elevada" .

De acordo com o RSI, a Emergência em Saúde Pública de Importância Internacional (ESPII) significa um evento extraordinário que constitui risco para a saúde pública de outros países, com potencial para propagação internacional e que, em geral, requer uma resposta internacional coordenada ${ }^{3}$. Segundo os conceitos definidos pelo RSI, "risco para a saúde pública significa a probabilidade de um evento que possa afetar adversamente a saúde de populações humanas" 3 .

O registro das informações referentes às potenciais emergências em saúde pública monitoradas pelo CIEVS/MS é realizado no Sistema de Monitoramento de Eventos em Saúde Pública (SIME), banco de dados paralelo, de base não nominal, com informações agregadas sobre os eventos, e de acesso restrito aos usuários cadastrados.

Considerando que a vigilância dos eventos de saúde pública é necessária para proporcionar o alerta ao sistema de vigilância do país ${ }^{9}$, o papel do CIEVS/MS é fundamental para a vigilância e resposta às emergências em saúde pública. Sendo assim, este artigo tem o objetivo de apresentar a avaliação das capacidades de vigilância do CIEVS/MS segundo o RSI, nas atribuições de detecção, avaliação e notificação das potenciais emergências em saúde pública de importância nacional e internacional.

\section{DESENVOLVIMENTO}

\section{MÉTODOS}

Foi desenvolvido um estudo de caso, de caráter avaliativo, sobre as capacidades de detecção, avaliação e notificação das potenciais emergências em saúde pública de importância nacional e internacional pelo CIEVS/MS, no período de janeiro a dezembro de 2012.

As fontes de dados para análise e julgamento dos indicadores foram os registros de mensagens 
eletrônicas do endereço institucional e os bancos de dados do SIME do CIEVS/MS, sendo realizada a comparação das informações descritivas dos eventos de saúde pública no SIME com os dados disponíveis nas mensagens do correio eletrônico.

A abordagem do estudo utilizou o modelo lógico de avaliação, de natureza qualitativa e quantitativa. Como estratégia de representação da funcionalidade de um programa por meio da síntese de seus principais componentes, o modelo lógico resume o mecanismo de funcionamento de um programa ou serviço em uma sequência de passos que une a estrutura, o processo e os resultados ${ }^{10,11}$.

Considerando que o modelo lógico permite definir um conjunto de indicadores para avaliação, tanto da estrutura quanto dos processos e dos resultados ${ }^{11}$, foram estabelecidos os indicadores para a avaliação dos processos de trabalho do CIEVS/MS. Os indicadores de processo foram definidos com a finalidade de interpretar os dados de modo contínuo e sistemático, além de possibilitar atualização e ajustes conforme necessário.

Para a elaboração das matrizes de análise e julgamento, foram identificados os componentes do processo de trabalho do CIEVS/MS envolvidos com a detecção, avaliação e notificação das potenciais emergências em saúde pública. As dimensões estratégicas foram selecionadas conforme o contexto institucional do CIEVS/MS, considerando-se os aspectos político, técnico e organizacional.

A dimensão política do trabalho do CIEVS/MS aborda as questões normatizadoras dos processos de detecção, avaliação e notificação dos eventos de saúde pública e das potenciais emergências em saúde pública. Relaciona-se ao papel estratégico da notificação e ao monitoramento das potenciais emergências em saúde pública de importância nacional e internacional, segundo regulamentado pelas leis, portarias e decretos nacionais, assim como pelas normativas internacionais.

A dimensão técnica refere-se ao desempenho dos profissionais envolvidos nos processos de trabalho do CIEVS/MS. Evidencia as responsabilidades das tarefas cotidianas, o registro das informações nos bancos de dados e o compartilhamento das informações relacionadas aos eventos de saúde pública e às emergências em saúde pública de importância nacional e internacional.

A dimensão organizacional está relacionada ao modo como a instituição se organiza para articular a vigilância das emergências em saúde pública. Esse aspecto envolve a forma de organização descentralizada da estratégia nos estados e nas capitais, bem como se refere à articulação intersetorial e interinstitucional no processo de compartilhamento das informações desenvolvido pelo CIEVS/ MS.

O primeiro componente do processo de trabalho do CIEVS/MS se refere à detecção, etapa em que são capturadas e/ou recebidas as informações a respeito das potenciais emergências em saúde pública de importância nacional e internacional. A detecção subdivide-se em duas vertentes: 
detecção ativa e detecção passiva. A detecção ativa representa a atividade de captura de rumores sobre eventos de saúde pública, reportados na mídia ou pela população, com a posterior confirmação da veracidade das informações. A detecção passiva trata dos eventos de saúde pública notificados ao CIEVS/MS pelos CIEVS/SES e CIEVS/SMS; unidades técnicas da Secretaria de Vigilância em Saúde do Ministério da Saúde (UT/SVS), das Secretarias Estaduais de Saúde (UT/SES) e das Secretarias Municipais de Saúde (UT/SMS); profissionais de saúde, serviços de saúde ou instituições parceiras do Ministério da Saúde, por meio do correio eletrônico, telefone de discagem direta gratuita ou formulário eletrônico de notificação disponível no site da Secretaria de Vigilância em Saúde (SVS).

O segundo componente consiste na avaliação, ou seja, análise da relevância em saúde pública dos eventos detectados ativa ou passivamente pelo CIEVS/MS. Corresponde ao processo de avaliação de risco dos eventos de saúde pública com base nos critérios do RSI: impacto de saúde pública; evento incomum ou inesperado; risco de propagação; interferência no fluxo de pessoas ou comércio de produtos. Esse componente do processo de trabalho é longitudinal em relação aos demais.

O terceiro componente caracteriza-se pela notificação, etapa de comunicação das informações qualificadas referentes aos eventos de saúde pública, avaliados segundo os critérios de classificação de risco, aos atores finalísticos da vigilância para as tomadas de decisão e as ações de resposta necessárias. Tal componente inclui a notificação das emergências em saúde pública de importância nacional e internacional aos atores nacionais e ao Ponto de Contato da OMS nas Américas, a Organização Pan-Americana da Saúde (OPAS), nos termos do RSI.

Para cada componente do processo de trabalho do CIEVS/MS foram construídos indicadores específicos, considerando-se cada uma das dimensões estratégicas. Para cada indicador foram definidos os padrões de análise e julgamento.

Todos os eventos notificados e monitorados pelo CIEVS/MS, no período de janeiro a dezembro de 2012, foram avaliados com base no modelo lógico e nas matrizes de análise e julgamento. A análise dos eventos monitorados foi ordenada segundo roteiro específico para observação direta dos processos de trabalho do CIEVS/MS.

A classificação das capacidades de detecção, avaliação e notificação das potenciais emergências em saúde pública de importância nacional e internacional pelo CIEVS/MS foi apresentada com base na predominância de indicadores classificados como Bom nas três dimensões estratégicas e nos três componentes do processo de trabalho.

As capacidades de detecção, avaliação e notificação das potenciais emergências em saúde pública pelo CIEVS/MS foram avaliadas, primeiramente, segundo componente do processo de trabalho e dimensão estratégica a partir da média de eventos monitorados com indicador Bom. Por fim, foi realizada a análise final das capacidades de vigilância do CIEVS/MS nas três dimensões estratégicas e nos três componentes, de acordo com a média de eventos monitorados com indicador 
Bom.

O estudo foi submetido ao Comitê de Ética em Pesquisa da Escola Nacional de Saúde Pública e aprovado segundo o parecer número 463.174, de 27 de novembro de 2013.

\section{RESULTADOS}

As matrizes de análise e julgamento de cada componente do processo de trabalho do CIEVS/ MS foram estruturadas com base nos três componentes e nas três dimensões estratégicas. A forma de cálculo dos indicadores segundo fontes de verificação dos dados é apresentada nas respectivas matrizes dos componentes (quadros 1, 2 e 3 ).

Quadro 1. Matriz de Análise e Julgamento: Componente Detecção

\begin{tabular}{|c|c|c|c|c|c|c|c|c|}
\hline \multicolumn{9}{|c|}{ Dimensões Estratégicas do Componente Detecção do Processo de Trabalho do CIEVS/MS } \\
\hline \multicolumn{3}{|c|}{ Política } & \multicolumn{3}{|c|}{ Técnica } & \multicolumn{3}{|c|}{ Organizacional } \\
\hline Indicador & \multicolumn{2}{|c|}{ Julgamento } & Indicador & \multicolumn{2}{|c|}{ Julgamento } & Indicador & \multicolumn{2}{|c|}{ Julgamento } \\
\hline $\begin{array}{c}\text { Contato do } \\
\text { CIEVS/MS } \\
\text { com CIEVS/ } \\
\text { SES e/ou UT/ } \\
\text { SVS para } \\
\text { verificação das } \\
\text { informações } \\
\text { no prazo de } 24 \\
\text { horas a partir } \\
\text { da detecção } \\
\text { ativa ou passiva }\end{array}$ & $\begin{array}{c}\text { Registro } \\
\text { do evento } \\
\text { de saúde } \\
\text { pública no } \\
\text { banco de } \\
\text { dados de } \\
\text { Notificações } \\
\text { do SIME }\end{array}$ & $\begin{array}{c}\text { Contato do } \\
\text { CIEVS/MS } \\
\text { com CIEVS/ } \\
\text { SES e/ou UT/ } \\
\text { SVS por meio } \\
\text { do correio } \\
\text { eletrônico no } \\
\text { prazo de } 24 \\
\text { horas a partir } \\
\text { da detecção } \\
\text { ativa ou } \\
\text { passiva }\end{array}$ & $\begin{array}{c}\text { Comunicação } \\
\text { do CIEVS/ } \\
\text { MS com } \\
\text { CIEVS/SES } \\
\text { e/ou UT/ } \\
\text { SVS para } \\
\text { atualização } \\
\text { das } \\
\text { informações } \\
\text { no prazo de } \\
72 \text { horas a } \\
\text { partir da } \\
\text { deteção ativa } \\
\text { ou passiva }\end{array}$ & $\begin{array}{c}\text { Registro de } \\
\text { atualizações } \\
\text { nos bancos } \\
\text { de dados de } \\
\text { Notificações } \\
\text { ou de } \\
\text { Eventos do } \\
\text { SIME }\end{array}$ & $\begin{array}{c}\text { Comunicação } \\
\text { do CIEVS/ } \\
\text { MS com } \\
\text { CIEVS/SES } \\
\text { e/ou UT/SVS } \\
\text { por meio } \\
\text { do correio } \\
\text { eletrônico } \\
\text { para } \\
\text { atualização } \\
\text { das } \\
\text { informações } \\
\text { no prazo } \\
\text { de } 72 \text { horas } \\
\text { a partir da } \\
\text { deteção } \\
\text { ativa ou } \\
\text { passiva }\end{array}$ & $\begin{array}{c}\text { Resposta } \\
\text { do CIEVS/ } \\
\text { SES e/ou } \\
\text { UT/SVS à } \\
\text { solicitação de } \\
\text { verificação } \\
\text { no prazo de } \\
24 \text { a } 48 \text { horas } \\
\text { a partir } \\
\text { do contato } \\
\text { inicial do } \\
\text { CIEVS/MS }\end{array}$ & $\begin{array}{l}\text { Não se } \\
\text { aplica às } \\
\text { variáveis } \\
\text { disponíveis } \\
\text { no SIME }\end{array}$ & $\begin{array}{c}\text { Resposta do } \\
\text { CIEVS/SES e/ } \\
\text { ou UT/SVS à } \\
\text { solicitação de } \\
\text { verificação do } \\
\text { CIEVS/MS por } \\
\text { meio do correio } \\
\text { eletrônico no } \\
\text { prazo de } 24 \text { a } 48 \\
\text { horas a partir do } \\
\text { contato inicial do } \\
\text { CIEVS/MS }\end{array}$ \\
\hline
\end{tabular}

Fonte: Elaborado pelos autores.

Quadro 2. Matriz de Análise e Julgamento: Componente Avaliação

\begin{tabular}{|c|c|c|c|c|c|c|c|c|}
\hline \multicolumn{9}{|c|}{ Dimensões Estratégicas do Componente Avaliação do Processo de Trabalho do CIEVS/MS } \\
\hline \multicolumn{3}{|c|}{ Política } & \multicolumn{3}{|c|}{ Técnica } & \multicolumn{3}{|c|}{ Organizacional } \\
\hline Indicador & \multicolumn{2}{|c|}{ Julgamento } & \multirow[b]{2}{*}{$\begin{array}{c}\text { Indicador } \\
\text { Avaliação } \\
\text { de risco } \\
\text { do evento } \\
\text { de saúde } \\
\text { pública } \\
\text { realizada } \\
\text { pelo CIEVS/ } \\
\text { MS em } \\
\text { articulação } \\
\text { com UT/SVS } \\
\text { de referência } \\
\text { do agravo }\end{array}$} & \multicolumn{2}{|c|}{ Julgamento } & Indicador & \multicolumn{2}{|c|}{ Julgamento } \\
\hline $\begin{array}{c}\text { Avaliação de } \\
\text { risco do evento } \\
\text { de saúde pública } \\
\text { discutida na } \\
\text { reunião semanal } \\
\text { do Comitê de } \\
\text { Monitoramento } \\
\text { de Eventos }\end{array}$ & $\begin{array}{l}\text { Registro da } \\
\text { avaliação de } \\
\text { risco do evento } \\
\text { de saúde } \\
\text { pública no } \\
\text { banco de dados } \\
\text { de Eventos do } \\
\text { SIME segundo a } \\
\text { reunião semanal } \\
\text { do Comitê de } \\
\text { Monitoramento } \\
\text { de Eventos }\end{array}$ & $\begin{array}{c}\text { Não se aplica } \\
\text { às informações } \\
\text { disponíveis } \\
\text { nas mensagens } \\
\text { eletrônicas } \\
\text { do endereço } \\
\text { institucional }\end{array}$ & & $\begin{array}{c}\text { Descrição } \\
\text { da } \\
\text { avaliação } \\
\text { de risco } \\
\text { do evento } \\
\text { de saúde } \\
\text { pública no } \\
\text { banco de } \\
\text { dados de } \\
\text { Eventos } \\
\text { do SIME }\end{array}$ & $\begin{array}{l}\text { Informação } \\
\text { sobre a } \\
\text { avaliação } \\
\text { de risco do } \\
\text { evento de } \\
\text { saúde pública } \\
\text { comunicada } \\
\text { por meio } \\
\text { do correio } \\
\text { eletrônico }\end{array}$ & $\begin{array}{c}\text { Fluxo de } \\
\text { informaç̃̃es } \\
\text { entre CIEVS/ } \\
\text { MS, CIEVS/ } \\
\text { SES e UT/ } \\
\text { SVS para } \\
\text { sustentar a } \\
\text { avaliação } \\
\text { de risco do } \\
\text { evento de } \\
\text { saúde pública }\end{array}$ & $\begin{array}{c}\text { Não se } \\
\text { aplica às } \\
\text { varíveis } \\
\text { disponíveis } \\
\text { no SIME }\end{array}$ & $\begin{array}{c}\text { Atualizações } \\
\text { referentes } \\
\text { à avaliação } \\
\text { de risco do } \\
\text { evento de } \\
\text { saúde pública } \\
\text { comunicadas } \\
\text { por meio } \\
\text { do correio } \\
\text { eletrônico }\end{array}$ \\
\hline
\end{tabular}

Fonte: Elaborado pelos autores. 
Quadro 3. Matriz de Análise e Julgamento: Componente Notificação

\begin{tabular}{|c|c|c|c|c|c|c|c|c|}
\hline \multicolumn{9}{|c|}{ Dimensões Estratégicas do Componente Notificação do Processo de Trabalho do CIEVS/MS } \\
\hline \multicolumn{3}{|c|}{ Política } & \multicolumn{3}{|c|}{ Técnica } & \multicolumn{3}{|c|}{ Organizacional } \\
\hline Indicador & \multicolumn{2}{|c|}{ Julgamento } & Indicador & \multicolumn{2}{|c|}{ Julgamento } & Indicador & \multicolumn{2}{|c|}{ Julgamento } \\
\hline $\begin{array}{c}\text { Notificação } \\
\text { do evento de } \\
\text { saúde pública } \\
\text { aos CIEVS/ } \\
\text { SES, UT/SVS } \\
\text { e parceiros no } \\
\text { prazo de } 24 \\
\text { horas a partir } \\
\text { da detecção } \\
\text { ativa ou } \\
\text { passiva }\end{array}$ & $\begin{array}{l}\text { Registro } \\
\text { do evento } \\
\text { de saúde } \\
\text { pública no } \\
\text { banco de } \\
\text { dados de } \\
\text { Notificações } \\
\text { do SIME }\end{array}$ & $\begin{array}{l}\text { Notificação } \\
\text { do evento de } \\
\text { saúde pública } \\
\text { aos CIEVS/ } \\
\text { SES, UT/SVS } \\
\text { e parceiros } \\
\text { por meio } \\
\text { do correio } \\
\text { eletrônico no } \\
\text { prazo de } 24 \\
\text { horas a partir } \\
\text { da detecção }\end{array}$ & $\begin{array}{c}\text { Envio das } \\
\text { atualizações } \\
\text { do evento de } \\
\text { saúde pública } \\
\text { no prazo de } \\
24 \text { horas a } \\
\text { partir do } \\
\text { recebimento } \\
\text { ou da captura }\end{array}$ & $\begin{array}{l}\text { Registro das } \\
\text { atualizações } \\
\text { do evento } \\
\text { de saúde } \\
\text { pública no } \\
\text { banco de } \\
\text { dados de } \\
\text { Notificações } \\
\text { do SIME }\end{array}$ & $\begin{array}{c}\text { Encaminhamento } \\
\text { das atualizações } \\
\text { do evento de } \\
\text { saúde pública } \\
\text { aos CIEVS/ } \\
\text { SES e UT/SVS } \\
\text { no prazo de } 24 \\
\text { horas a partir do } \\
\text { recebimento ou } \\
\text { da captura }\end{array}$ & $\begin{array}{c}\text { Resposta da } \\
\text { UT/SVS e/ } \\
\text { ou CIEVS/ } \\
\text { SES no prazo } \\
\text { de } 48 \text { horas } \\
\text { à notificação } \\
\text { do evento de } \\
\text { saúde pública } \\
\text { pelo CIEVS/ } \\
\text { MS }\end{array}$ & $\begin{array}{c}\text { Não se } \\
\text { aplica às } \\
\text { variáveis } \\
\text { disponíveis } \\
\text { no SIME }\end{array}$ & $\begin{array}{c}\text { Resposta da } \\
\text { UT/SVS e/ } \\
\text { ou CIEVS/ } \\
\text { SES no } \\
\text { prazo de } \\
48 \text { horas à } \\
\text { notificação } \\
\text { do evento } \\
\text { de saúde } \\
\text { pública pelo } \\
\text { CIEVS/MS }\end{array}$ \\
\hline
\end{tabular}

Fonte: Elaborado pelos autores.

\section{Análise dos indicadores}

Com base nas matrizes de análise e julgamento dos processos de trabalho do CIEVS/MS, foi desenvolvida a avaliação dos 58 eventos de saúde pública de importância nacional e internacional monitorados pelo serviço no período de janeiro a dezembro de 2012. Aavaliação de cada componente do processo de trabalho do CIEVS/MS vinculado com a detecção, avaliação e notificação dos eventos de saúde pública foi expressa de acordo com os achados obtidos a partir da análise dos indicadores nas três dimensões estratégicas.

Para cada indicador foram definidos os padrões de análise e julgamento, distribuídos nas seguintes categorias: Bom - indicador contemplado integralmente; Regular - indicador contemplado parcialmente; Insuficiente - indicador contemplado de forma incipiente; Crítico - indicador não teve nenhum requisito contemplado. Os resultados da análise dos indicadores referentes a cada evento de saúde pública são apresentados no quadro 4.

Quadro 4. Eventos de saúde pública monitorados segundo análise dos indicadores (continua)

\begin{tabular}{|c|c|c|c|c|c|c|c|c|c|c|}
\hline \multirow{2}{*}{$\begin{array}{c}\text { Eventos } \\
\text { de saúde } \\
\text { pública }\end{array}$} & \multicolumn{6}{|c|}{ COMPONENTES DO PROCESSO DE TRABALHO DO CIEVS/MS } \\
\cline { 2 - 11 } & \multicolumn{7}{|c|}{ Deteç̧ão } & \multicolumn{7}{c|}{ Avaliação } & \multicolumn{3}{c|}{ Notificação } \\
\cline { 2 - 10 } & Política & Técnica & Organizacional & Política & Técnica & Organizacional & Política & Técnica & Organizacional \\
\hline $\begin{array}{c}\text { Síndrome } \\
\text { Respiratória } \\
\text { Aguda Grave }\end{array}$ & Bom & Regular & Bom & Bom & Bom & Bom & Bom & Regular & Crítico \\
\hline Raiva humana & Bom & Regular & Bom & Bom & Bom & Bom & Bom & Regular & Bom \\
\hline Malária & Bom & Bom & Bom & Bom & Bom & Bom & Bom & Regular & Bom \\
\hline $\begin{array}{c}\text { Síndrome } \\
\text { Respiratória } \\
\text { Aguda Grave }\end{array}$ & Bom & Bom & Bom & Bom & Bom & Bom & Bom & Bom & Bom \\
\hline $\begin{array}{c}\text { Febre Maculosa } \\
\text { Brasileira }\end{array}$ & Crítico & Insuficiente & Crítico & Bom & Regular & Crítico & Crítico & Crítico & Crítico \\
\hline Raiva humana & Bom & Bom & Crítico & Bom & Bom & Bom & Bom & Regular & Crítico \\
\hline Raiva humana & Bom & Insuficiente & Bom & Bom & Bom & Bom & Regular & Regular & Bom \\
\hline
\end{tabular}




\begin{tabular}{|c|c|c|c|c|c|c|c|c|c|}
\hline $\begin{array}{c}\text { Meningite } \\
\text { meningocócica }\end{array}$ & Regular & Insuficiente & Bom & Bom & Bom & Crítico & Regular & Crítico & Bom \\
\hline Febre amarela & Regular & Insuficiente & Crítico & Bom & Regular & Bom & Regular & Regular & Crítico \\
\hline $\begin{array}{l}\text { Botulismo } \\
\text { alimentar }\end{array}$ & Bom & Bom & Bom & Bom & Bom & Bom & Bom & Regular & Bom \\
\hline $\begin{array}{c}\text { Meningite } \\
\text { meningocócica }\end{array}$ & Bom & Regular & Bom & Bom & Bom & Bom & Bom & Regular & Bom \\
\hline $\begin{array}{c}\text { Febre Maculosa } \\
\text { Brasileira }\end{array}$ & Bom & Regular & Bom & Bom & Bom & Bom & Bom & Crítico & Bom \\
\hline $\begin{array}{c}\text { Etiologia } \\
\text { indeterminada }\end{array}$ & Bom & Regular & Bom & Bom & Bom & Bom & Bom & Regular & Bom \\
\hline $\begin{array}{l}\text { Meningite } \\
\text { meningocócica }\end{array}$ & Regular & Bom & Bom & Bom & Bom & Bom & Regular & Regular & Bom \\
\hline $\begin{array}{l}\text { Intoxicação } \\
\text { exógena }\end{array}$ & Bom & Bom & Bom & Bom & Bom & Bom & Bom & Regular & Bom \\
\hline Raiva humana & Bom & Regular & Bom & Bom & Bom & Bom & Bom & Regular & Bom \\
\hline Síndrome gripal & Bom & Regular & Bom & Bom & Bom & Bom & Bom & Bom & Bom \\
\hline Chikungunya & Bom & Bom & Bom & Bom & Bom & Bom & Bom & Regular & Bom \\
\hline $\begin{array}{c}\text { Doença } \\
\text { Diarreica } \\
\text { Aguda }\end{array}$ & Regular & Regular & Bom & Bom & Bom & Bom & Regular & Regular & Bom \\
\hline $\begin{array}{c}\text { Doença } \\
\text { Diarreica } \\
\text { Aguda }\end{array}$ & Regular & Bom & Bom & Bom & Bom & Bom & Regular & Regular & Bom \\
\hline $\begin{array}{l}\text { Intoxicação } \\
\text { exógena }\end{array}$ & Bom & Insuficiente & Crítico & Bom & Bom & Bom & Bom & Bom & Crítico \\
\hline $\begin{array}{c}\text { Síndrome } \\
\text { Respiratória } \\
\text { Aguda Grave }\end{array}$ & Bom & Insuficiente & Crítico & Bom & Bom & Bom & Bom & Bom & Crítico \\
\hline Leptospirose & Bom & Bom & Bom & Bom & Bom & Bom & Bom & Bom & Bom \\
\hline $\begin{array}{l}\text { Leishmaniose } \\
\text { Tegumentar } \\
\text { Americana }\end{array}$ & Bom & Bom & Bom & Bom & Bom & Bom & Bom & Bom & Bom \\
\hline $\begin{array}{l}\text { Intoxicação } \\
\text { exógena }\end{array}$ & Bom & Bom & Bom & Bom & Bom & Bom & Bom & Bom & Bom \\
\hline $\begin{array}{l}\text { Botulismo } \\
\text { alimentar }\end{array}$ & Bom & Regular & Crítico & Bom & Bom & Bom & Bom & Regular & Crítico \\
\hline $\begin{array}{l}\text { Doença } \\
\text { Diarreica } \\
\text { Aguda }\end{array}$ & Bom & Insuficiente & Bom & Bom & Bom & Bom & Bom & Regular & Bom \\
\hline $\begin{array}{l}\text { Botulismo } \\
\text { alimentar }\end{array}$ & Bom & Insuficiente & Crítico & Bom & Bom & Bom & Bom & Bom & Crítico \\
\hline Desastre natural & Crítico & Regular & Crítico & Bom & Bom & Bom & Crítico & Regular & Crítico \\
\hline Desastre natural & Crítico & Insuficiente & Crítico & Bom & Regular & Crítico & Crítico & Crítico & Crítico \\
\hline
\end{tabular}

Fonte: Elaborado pelos autores.

Quadro 4. Eventos de saúde pública monitorados segundo análise dos indicadores (conclusão)

\begin{tabular}{|c|c|c|c|c|c|c|c|c|c|c|}
\hline \multirow{2}{*}{$\begin{array}{c}\text { Eventos } \\
\text { de saúde } \\
\text { pública }\end{array}$} & \multicolumn{7}{|c|}{ COMPONENTES DO PROCESSO DE TRABALHO DO CIEVS/MS } \\
\cline { 2 - 11 } & \multicolumn{7}{|c|}{ Detecção } & \multicolumn{7}{c|}{ Avaliação } & \multicolumn{3}{c|}{ Notificação } \\
\cline { 2 - 11 } & Política & Técnica & Organizacional & Política & Téenica & Organizacional & Política & Técnica & Organizacional \\
\hline Desastre natural & Bom & Bom & Bom & Bom & Regular & Bom & Bom & Regular & Bom \\
\hline Desastre natural & Bom & Bom & Bom & Bom & Regular & Bom & Bom & Bom & Bom \\
\hline Desastre natural & Bom & Bom & Bom & Bom & Regular & Bom & Bom & Regular & Bom \\
\hline Sarampo & Regular & Regular & Bom & Bom & Bom & Insuficiente & Regular & Regular & Bom \\
\hline Sarampo & Bom & Bom & Bom & Bom & Bom & Bom & Bom & Regular & Bom \\
\hline Chikungunya & Regular & Regular & Bom & Bom & Regular & Bom & Regular & Regular & Bom \\
\hline
\end{tabular}

Continua 
Continuação

\begin{tabular}{|c|c|c|c|c|c|c|c|c|c|}
\hline Meningite viral & Regular & Regular & Crítico & Bom & Regular & Bom & Regular & Regular & Insuficiente \\
\hline Cólera & Regular & Bom & Bom & Bom & Regular & Bom & Regular & Regular & Bom \\
\hline $\begin{array}{c}\text { Doença } \\
\text { Diarreica } \\
\text { Aguda }\end{array}$ & Regular & Bom & Bom & Bom & Bom & Bom & Regular & Regular & Bom \\
\hline Coqueluche & Regular & Bom & Bom & Bom & Bom & Bom & Regular & Regular & Bom \\
\hline $\begin{array}{l}\text { Botulismo } \\
\text { alimentar }\end{array}$ & Regular & Bom & Bom & Bom & Bom & Bom & Regular & Regular & Bom \\
\hline Febre amarela & Regular & Regular & Crítico & Bom & Bom & Insuficiente & Regular & Crítico & Crítico \\
\hline $\begin{array}{c}\text { Meningite } \\
\text { meningocócica }\end{array}$ & Bom & Bom & Bom & Bom & Bom & Bom & Bom & Regular & Bom \\
\hline Sarampo & Regular & Regular & Insuficiente & Bom & Bom & Crítico & Regular & Crítico & Crítico \\
\hline Síndrome gripal & Bom & Bom & Bom & Bom & Bom & Bom & Bom & Regular & Bom \\
\hline $\begin{array}{l}\text { Intoxicação } \\
\text { exógena }\end{array}$ & Regular & Bom & Bom & Bom & Bom & Bom & Regular & Regular & Bom \\
\hline Raiva humana & Regular & Regular & Insuficiente & Bom & Bom & Bom & Crítico & Regular & Insuficiente \\
\hline Malária & Regular & Regular & Bom & Bom & Regular & Insuficiente & Regular & Regular & Bom \\
\hline Síndrome gripal & Regular & Bom & Bom & Bom & Bom & Bom & Regular & Regular & Bom \\
\hline $\begin{array}{c}\text { Doença de } \\
\text { Chagas Aguda }\end{array}$ & Regular & Regular & Crítico & Bom & Regular & Crítico & Regular & Regular & Crítico \\
\hline Hantavirose & Regular & Regular & Crítico & Bom & Regular & Crítico & Regular & Regular & Crítico \\
\hline Febre amarela & Regular & Bom & Bom & Bom & Bom & Bom & Regular & Regular & Bom \\
\hline Raiva humana & Regular & Bom & Bom & Bom & Bom & Insuficiente & Regular & Regular & Bom \\
\hline $\begin{array}{c}\text { Febre Maculosa } \\
\text { Brasileira }\end{array}$ & Regular & Bom & Bom & Bom & Bom & Bom & Regular & Regular & Bom \\
\hline Hepatite A & Bom & Bom & Bom & Bom & Bom & Bom & Bom & Regular & Bom \\
\hline $\begin{array}{c}\text { Etiologia } \\
\text { indeterminada }\end{array}$ & Bom & Bom & Bom & Bom & Bom & Bom & Bom & Regular & Bom \\
\hline $\begin{array}{c}\text { Meningite } \\
\text { meningocócica }\end{array}$ & Crítico & Insuficiente & Crítico & Bom & Regular & Crítico & Crítico & Crítico & Crítico \\
\hline $\begin{array}{c}\text { Doença } \\
\text { Diarreica } \\
\text { Aguda }\end{array}$ & Bom & Regular & Bom & Bom & Bom & Bom & Bom & Bom & Bom \\
\hline
\end{tabular}

Fonte: Elaborado pelos autores.

\section{Componente Detecção}

A análise da dimensão política demonstrou que, do total de eventos, 31 (53\%) apresentaram o indicador classificado como Bom, e 23 eventos (40\%) indicador Regular.

A dimensão técnica permitiu avaliar as rotinas de trabalho do CIEVS/MS vinculadas à obtenção dos dados e ao registro de atualizações dos eventos de saúde pública monitorados. A avaliação identificou 28 eventos (48\%) com indicador Bom e 20 eventos (34\%) com indicador Regular.

A dimensão organizacional permitiu avaliar a articulação entre o CIEVS/MS, os CIEVS/SES e as UT/SVS no processo de comunicação referente aos eventos de saúde pública de importância nacional e internacional. Constatou-se que o indicador organizacional foi Bom em 42 eventos (72\%). Foram classificados, por sua vez, 14 eventos (24\%) com indicador Crítico.

Com base na análise detalhada por meio das mensagens eletrônicas, observou-se que, dentre os eventos não contemplados ou incipientes, nove (56\%) foram comunicados pelo CIEVS/MS aos 
CIEVS/SES e UT/SVS às sextas-feiras ou durante os finais de semana. Tal resultado demonstra que a acessibilidade ininterrupta dos serviços de vigilância das emergências em saúde pública consiste em fator influenciador do processo de trabalho.

\section{Componente Avaliação}

A análise da dimensão política refletiu o processo sistemático de avaliação de risco dos eventos de saúde pública nas reuniões semanais do Comitê de Monitoramento de Eventos (CME), além da padronização da nomenclatura de Risco para a Saúde Pública Nacional (RSPN) segundo o RSI. Considerando-se que a classificação RSPN passou a ser utilizada para demonstrar que os eventos de saúde pública monitorados pelo CIEVS/MS representam riscos potenciais à saúde pública nacional, todos os eventos foram contemplados nessa dimensão e o indicador foi Bom em 100\%.

A análise da dimensão técnica demonstrou o trabalho articulado do CIEVS/MS com as demais unidades técnicas da SVS para realizar a avaliação de risco dos eventos de saúde pública. Foram classificados com indicador Bom 45 eventos (78\%). Os 13 eventos restantes (22\%) obtiveram indicador Regular.

Como a dimensão organizacional está relacionada ao modo como a instituição se organiza para articular a vigilância e a classificação de risco das emergências em saúde pública, o indicador buscou avaliar a continuidade do processo de avaliação de risco dos eventos monitorados. Observou-se que 47 eventos (81\%) foram classificados com indicador Bom.

\section{Componente Notificação}

A dimensão política relaciona-se ao papel regulamentado do CIEVS/MS de congregar mecanismos de comunicação avançados para o monitoramento das emergências em saúde pública nos âmbitos nacional e internacional. Portanto, buscou avaliar a oportunidade de notificação dos eventos de saúde pública pelo CIEVS/MS aos CIEVS/SES, UT/SVS e demais atores. Observou-se que 30 eventos (52\%) foram classificados com indicador Bom e 23 eventos (40\%) apresentaram indicador Regular.

A análise da dimensão técnica permitiu observar a atualização das informações nos bancos de dados do SIME, além de possibilitar a verificação da resposta e do encaminhamento oportuno das informações pelo CIEVS/MS. Apenas 10 eventos (17\%) foram classificados com indicador Bom. A classificação Regular foi apresentada em 41 eventos (71\%) e Crítico em 7 eventos (12\%).

A dimensão organizacional, por fim, permitiu avaliar o trabalho do CIEVS/MS em articulação com as demais unidades técnicas da SVS e CIEVS/SES. Verificou-se que 41 eventos (71\%) apresentaram indicador Bom. Entretanto, 15 eventos (26\%) resultaram em indicador Crítico.

Assim como observado na dimensão organizacional do componente detecção, a resposta oportuna ao CIEVS/MS depende do funcionamento e acessibilidade dos CIEVS/SES e UT/SVS durante os períodos noturnos, finais de semana e feriados. Nesse contexto, dentre os eventos cujo indicador foi classificado como Insuficiente ou Crítico, nove (53\%) foram notificados às sextasfeiras ou nos finais de semana. 


\section{Capacidades de vigilância}

As capacidades de detecção, avaliação e notificação das potenciais emergências em saúde pública de importância nacional e internacional pelo CIEVS/MS foram classificadas com base na predominância de indicadores classificados como Bom nas três dimensões estratégicas e nos três componentes do processo de trabalho. Assim, as possibilidades de classificação foram definidas conforme as seguintes categorias: Adequada - acima de 70\% de indicadores classificados como Bom nas três dimensões e nos três componentes; Parcialmente adequada ou Parcial - entre $50 \%$ e $70 \%$ de indicadores classificados como Bom nas três dimensões e nos três componentes; Insuficiente - entre $30 \%$ e $50 \%$ de indicadores classificados como Bom nas três dimensões e nos três componentes; Incipiente - abaixo de 30\% de indicadores classificados como Bom nas três dimensões e nos três componentes.

Considerando-se a relevância de cada dimensão estratégica e cada componente do processo de trabalho, não houve diferença de ponderação dos fatores para a definição da predominância dos indicadores. Sendo assim, a prevalência dos indicadores classificados como Bom foi baseada na média aritmética simples do número de eventos com indicador Bom nas três dimensões estratégicas e nos três componentes do processo de trabalho.

Os resultados da análise das capacidades de vigilância do CIEVS/MS são apresentados na tabela 1.

Tabela 1. Capacidades de vigilância do CIEVS/MS segundo distribuição dos eventos com indicador Bom por dimensão estratégica e componente do processo de trabalho

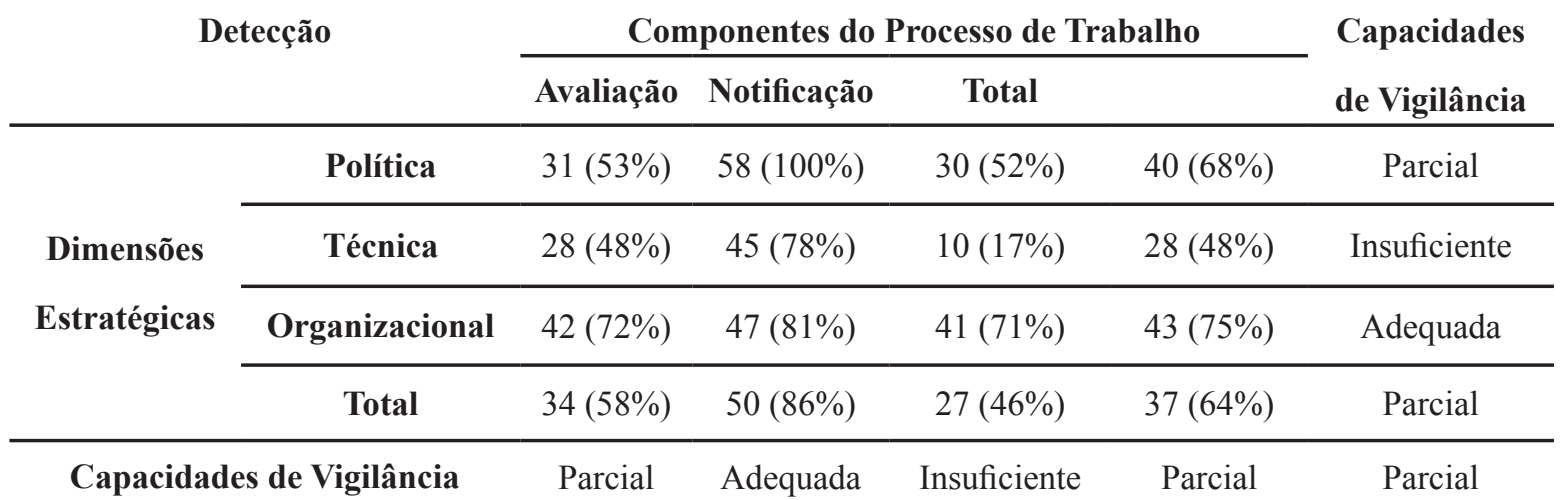

Fonte: Elaborado pelos autores.

A análise das capacidades de detecção, avaliação e notificação foi realizada segundo componente do processo de trabalho e o resultado final foi considerado a partir da média aritmética simples do número de eventos com indicador Bom distribuídos nas três dimensões estratégicas.

No componente deteç̧ão, a capacidade do CIEVS/MS foi classificada como parcialmente adequada. No componente avaliação, por outro lado, a capacidade do CIEVS/MS foi classificada como adequada. Por fim, no componente notificação, a capacidade do CIEVS/MS foi classificada como insuficiente. 
As capacidades de detecção, avaliação e notificação do CIEVS/MS também foram analisadas de acordo com as dimensões estratégicas e o resultado final foi considerado a partir da média aritmética simples do número de eventos com indicador Bom distribuídos nos componentes do processo de trabalho.

Na dimensão política, a capacidade do CIEVS/MS foi classificada como parcialmente adequada. De acordo com a dimensão técnica, a capacidade do CIEVS/MS foi classificada como insuficiente. $\mathrm{Na}$ dimensão organizacional, por sua vez, a capacidade do CIEVS/MS foi classificada como adequada.

As capacidades de vigilância do CIEVS/MS foram calculadas de acordo com a capacidade total de detecção, avaliação e notificação nos componentes do processo de trabalho e nas dimensões estratégicas. Segundo os componentes do processo de trabalho, a capacidade total do CIEVS/ MS foi classificada como parcialmente adequada. De modo similar, de acordo com as dimensões estratégicas, a capacidade total do CIEVS/MS foi classificada como parcialmente adequada.

Portanto, as capacidades de vigilância do CIEVS/MS foram avaliadas como parcialmente adequadas, o que corresponde ao critério de 50\% a 70\% de indicadores classificados como Bom nas três dimensões estratégicas e nos três componentes do processo de trabalho.

\section{CONCLUSÃO}

A avaliação das capacidades de vigilância do CIEVS/MS contribui para o processo de monitoramento das ações de vigilância e resposta às emergências em saúde pública no Brasil, assim como permite subsidiar o gerenciamento dos processos de trabalho relacionados à vigilância baseada em eventos.

Os resultados da análise dos indicadores de processo demonstram o diagnóstico situacional da estratégia de vigilância das emergências em saúde pública no âmbito da SVS. Nesse contexto, revelam as potencialidades e as fragilidades dos processos de trabalho do CIEVS/MS e fundamentam a importância da organização das atividades para o adequado cumprimento dos objetivos do serviço segundo o RSI.

As potencialidades observadas centram-se no componente avaliação e na dimensão organizacional. Tal diagnóstico revela que a atuação do CIEVS/MS se destaca no desenvolvimento da avaliação de risco dos eventos de saúde pública e na articulação efetiva com os atores envolvidos na vigilância e resposta às emergências em saúde pública.

Por outro lado, foram verificadas fragilidades nos componentes detecção e notificação, bem como nas dimensões política e técnica. Esses resultados significam que há necessidade de aprimoramento dos seguintes processos de trabalho: registro adequado das informações nos bancos de dados; notificação e comunicação rápida dos eventos de saúde pública; atualização dos eventos monitorados; encaminhamento oportuno das informações.

Usualmente, os sistemas de vigilância baseada em indicadores são avaliados de acordo com os atributos qualitativos (simplicidade, flexibilidade, estabilidade, qualidade dos dados e aceitabilidade), 
atributos quantitativos (sensibilidade, representatividade, oportunidade e valor preditivo positivo) e utilidade ${ }^{12}$. No entanto, diante da falta de referência internacional para avaliação dos sistemas de vigilância baseada em eventos, a aplicação do modelo lógico na análise dos processos de trabalho revelou-se como método importante para avaliação do serviço responsável pela vigilância das emergências em saúde pública no país.

Considerando-se que a inteligência epidemiológica resulta da confluência das informações obtidas a partir das estratégias de vigilância baseada em indicadores e vigilância baseada em eventos $^{13,14}$, a vigilância das emergências em saúde pública poderia ser aprimorada com base na utilização dos dados gerados na rotina da vigilância epidemiológica, de modo a facilitar a confirmação da veracidade das informações e produzir alertas para a identificação oportuna de potenciais emergências em saúde pública.

Ademais, sabendo-se que a estratégia de vigilância baseada em eventos é preconizada pela OMS com a finalidade de alerta e resposta rápida às emergências em saúde pública ${ }^{15}$ e que tal estratégia foi aplicada durante os Jogos Olímpicos de Londres 2012, sendo avaliada como útil, aceitável, estável e confiável ${ }^{16}$, recomenda-se que os processos de trabalho do CIEVS/MS sejam sistematizados e fortalecidos para a adequada vigilância das emergências em saúde pública no Brasil, inclusive, para o monitoramento das potenciais emergências em saúde pública durante os eventos de massa ${ }^{17}$, como a Copa do Mundo e os Jogos Olímpicos.

\section{Contribuição dos autores}

A primeira autora trabalhou na concepção, pesquisa, metodologia e redação. Os demais autores trabalharam na orientação, revisão crítica, análise e delineamento final. O artigo resulta da dissertação apresentada à Escola Nacional de Saúde Pública Sergio Arouca para obtenção do grau de Mestre em Saúde Pública.

\section{REFERÊNCIAS BIBLIOGRÁFICAS}

1. Waldman EA. Vigilância como prática de saúde pública. In: Campos GWS et al, org. Tratado de saúde coletiva. Rio de Janeiro: Fiocruz; 2006. p. 487-528.

2. Ministério da Saúde. Secretaria de Vigilância em Saúde. Guia de vigilância em saúde. 1. ed. Brasília: Ministério da Saúde, 2014.

3. World Health Organization. International Health Regulations (2005). 2nd ed. Geneve: WHO, 2008 .

4. Carmo EH. O Regulamento Sanitário Internacional e as oportunidades para fortalecimento do sistema de vigilância em saúde. Recife, PE: Portal DSS Nordeste; 2013 [acesso em 22 jun 2016]. Disponível em: http://dssbr.org/site/opinioes/o-regulamento-sanitario-internacional-e-asoportunidades-para-fortalecimento-do-sistema-de-vigilancia-em-saude/.

5. Brasil. Ministério da Saúde. Portaria n ${ }^{\circ}$ 1.865, de 10 de agosto de 2006. Estabelece a Secretaria de Vigilância em Saúde para o Regulamento Sanitário Internacional (2005) junto à Organização 
Mundial de Saúde. Diário Oficial da União 11 ago 2006.

6. Brasil. Ministério da Saúde. Secretaria de Vigilância em Saúde. Portaria $n^{\circ} 30$, de 7 de julho de 2005. Institui o Centro de Informações Estratégicas em Vigilância em Saúde, define suas atribuições, composição e coordenação. Diário Oficial da União 7 jul 2005.

7. World Health Organization. Western Pacific Region. A Guide to Establishing Event-based Surveillance. Genebra: WHO; 2008 [acesso em 22 jun 2016]. Disponível em: http://www.wpro. who.int/emerging_diseases/documents/docs/eventbasedsurv.pdf.

8. Brasil. Presidência da República. Casa Civil. Decreto nº 7.616, de 17 de novembro de 2011. Dispõe sobre a declaração de Emergência em Saúde Pública de Importância Nacional - ESPIN e institui a Força Nacional do Sistema Único de Saúde - FN-SUS. Diário Oficial da União 18 nov 2011.

9. Carmo EH, Penna G, Oliveira WK. Emergências de saúde pública: conceito, caracterização, preparação e resposta. Estudos avançados 2008; 22(64):19-32.

10. Contandriopoulos AP et al. A avaliação na área da saúde: conceitos e métodos. In: Hartz ZMA, org. Avaliação em Saúde. Dos modelos conceituais à prática na análise de implantação de programas. Rio de Janeiro: Ed. Fiocruz, 1999. p. 29-47.

11. Centers for Disease Control and Prevention. Framework for program evaluation in public health. MMWR 1999 [acesso em 22 jun 2016];48(RR11):1-40. Disponível em: https://www.cdc. gov/mmwr/PDF/rr/rr4811.pdf.

12. Centers for Disease Control and Prevention. Updated Guidelines for Evaluating Public Health Surveillance Systems. MMWR 2001 [acesso em 22 jun 2016];50(RR13):1-35. Disponível em: http://www.cdc.gov/mmwr/preview/mmwrhtml/rr5013a1.htm.

13. Paquet C, Coulombier D, Kaiser R, Ciotti M. Epidemic intelligence: a new framework for strengthening disease surveillance in Europe. Euro Surveill. 2006 [acesso em 22 jun 2016];11(12):pii=665. Disponível em: $\quad$ http://www.eurosurveillance.org/ViewArticle. aspx?ArticleId=665.

14. World Health Organization. WHO Technical consultation on event-based surveillance. Meeting report. Genebra: WHO, 2013 [acesso em 22 jun 2016]. Disponível em: http://www. episouthnetwork.org/sites/default/files/meeting_report_ebs_march_2013 final.pdf.

15. World Health Organization. Early detection, assessment and response to acute public health events: Implementation of Early Warning and Response with a focus on Event-Based Surveillance. Genebra: WHO, 2014 [acesso em 22 jun 2016]. Disponível em: http://apps.who.int/ iris/bitstream/10665/112667/1/WHO_HSE_GCR_LYO_2014.4_eng.pdf.

16. Severi E, Kitching A, Crook PD. Evaluation of the Health Protection Event-Based Surveillance 
for the London 2012 Olympic and Paralympic Games. Euro Surveill. 2014 [acesso em 22 jun 2016]; 19(24):pii=20832. Disponível em: http://www.eurosurveillance.org/ViewArticle.aspx?ArticleId=20832.

17. Brasil. Ministério da Saúde. Portaria $n^{0} 1.139$, de 10 de junho de 2013. Define, no âmbito do Sistema único de Saúde (SUS), as responsabilidades das esferas de gestão e estabelece as Diretrizes Nacionais para Planejamento, Execução e Avaliação das Ações de Vigilância e Assistência à Saúde em Eventos de Massa. Diário Oficial da União 11 jun 2013; Seção1, p. 22-24.

Artigo apresentado em 15-08-15

Artigo aprovado em 21-02-16 Artigo publicado no sistema em 22-03-16 